\title{
Pill-swallowing - a bitter experience for some despite the ease of the swallow
}

\author{
J Seedat, PhD Speech Pathology; A Zayannakis, BA Speech Pathology and Audiology \\ Department of Speech Therapy, Faculty of Humanities, University of the Witwatersrand, Johannesburg, South Africa \\ Corresponding author: J Seedat (Jaishika.seedat@wits.ac.za)
}

\begin{abstract}
Background. The need for pills in the form of analgesics, psychotropic medication, chronic medication or vitamins and supplements is gaining momentum globally. In developing contexts, social circumstances, the burden of disease, violence and poor lifestyles and eating habits all contribute to reliance on medication. Despite this knowledge, research that captures pill adherence, pill swallowing, coping by individuals in general practice and pill modification by the general public has been minimal in South Africa, a developing country.

Objectives. To address this gap by addressing the question: what are the experiences of adults in general practice who take pills? The objectives were to document the pill-swallowing experience of the adult, to identify variables that positively and negatively affected the swallowing process and to understand the perceptions of pill-swallowing, and what informed these perceptions.

Methods. An exploratory qualitative research design was used. Using convenience and snowball sampling, 73 participants were recruited, and consented to participate. They completed a researcher-developed questionnaire. Thematic content analysis and descriptive statistics were used for analysis.

Results. The results indicated that $67 \%$ of the participants included in the study found the swallowing process to be easy, whereas $33 \%$ found it to be unpleasant. A total of 56 participants described how the properties of the pill affected the swallow. They had a more pleasurable experience when taking small, coated capsules rather than large, uncoated tablets.

Conclusion. This study showed how pill properties could cause symptoms of dysphagia. It increases our understanding of the way that individuals from the general population who do not present with dysphagia may still experience problems with swallowing when it involves pills.
\end{abstract}

South Afr J Pub Health 2020;4(2):41-46. https://doi.org/10.7196/SHS.2020.v4.i2.108

Pill-swallowing is fast becoming a daily routine for many adults worldwide. The increasing incidence of chronic conditions, and an increasing awareness of the need for health and fitness, facilitating longevity and alleviation of illness and ailments, have gained momentum over the last century. ${ }^{[1]}$ As a result, more and more adults require prescription medication, and also over-the-counter medications in the way of acute medication for common ailments such as pain and inflammation, and off-the-shelf pills such as vitamins and diet supplements, all of which contribute to the medical and mental wellbeing of the individual. While there are merits in differentiating between those who do not have a choice, and have to take pills for medical reasons, and those who may be able to opt out of a pill regimen if required to, ultimately both cohorts perceive a need for the pill, whether for direct medical benefit or related mental and overall health wellbeing. Developed contexts have published data on medication use and dispensation. ${ }^{[2]}$ In the USA, the National Centre for Health Statistics says that $48.9 \%$ of the population had used at least one prescription pill in a 30-day period from 2011 - 2014. ${ }^{[3]}$ Furthermore, 73.9\% of visits to a physician in the USA involved medication dispensing. ${ }^{[4]}$ These figures raise questions about how these individuals coped with the medication regimen, and whether their regimens were completed as required. Such data are lacking in South Africa ( $\mathrm{SA}$ ), prompting the need to explore the pill-swallowing routine, challenges, coping strategies, pill acceptability and overall pill-swallowing experience.

In SA, the health and medical needs of the population are multilayered and diverse, and compounded by diverse living conditions, socioeconomic status, access and levels of education. SA faces a quadruple burden of disease, of which three - HIV/AIDS and tuberculosis, non-communicable diseases, and violence and injury - have direct implications for pill dispensing to adults. Power differentials between the doctor and patient are common across the country. ${ }^{[5]}$ This is significant, as individuals do not disclose to the doctor their difficulty swallowing pills, or the doctor assumes that pill-swallowing is easy for an adult, and does not query this prior to writing the script ${ }^{[6]}$ While research in Norway and the USA documents $26 \%$ and $40 \%$ of adults presenting with pill swallowing difficulties, respectively, such data are lacking in SA. ${ }^{[7,8]}$ Given that people are living longer owing to advances in the field of medicine, it is worrying that there may be a growing cohort of normally ageing 


\section{RESEARCH}

individuals who may find swallowing pills of different consistencies, sizes and shapes increasingly difficult as their functional capabilities change. ${ }^{[9]}$

Concerns arise as to whether individuals who are required to take pills are, firstly, compliant and adhere, and complete their pill regimen, or whether they alter the pills' physical properties to make them easier to swallow. To facilitate the efficacy of pills, swallowing them correctly is a necessity. The intended benefit of the pill is only realised if it is taken in its intended form and delivered to the site of dissolution. By understanding the experience from the perspective of the individual, one may be able to make causal links between pill regimen completion rates, pill-swallowing adherence, prevention of comorbidities, resolution of the underlying medical aetiology and so forth. The current study aimed to explore the swallowing experience from the perspective of the individual required to take the pill.

\section{Methodology}

The presentstudy aimed to document the pill-swallowing experience of the adult, identify variables that positively and negatively affected the swallowing process and understand perceptions of pill-swallowing and what informed these perceptions.

An exploratory qualitative research design was used. The participants were individuals from the general SA population who either take or have taken pills (for medical or health reasons, e.g. antibiotics, vitamins and/or supplements) regularly (either medication taken daily, or medication taken once a day, for a minimum of 5 days). Non-probability, convenience and snowball sampling were used to recruit participants.

As inclusion criteria, participants were individuals who were currently taking or who had previously taken pills on a regular basis, i.e. daily or every day for a minimum of 5 days. The individuals did not need to be taking the pill(s) at the time of data collection. Both male and females $>18$ years old were included. Participants had to have a conversational level of English.

Individuals who had been diagnosed with dysphagia previously were excluded. Individuals with a diagnosed neurological condition (such as a stroke or a traumatic brain injury and a history of dysphagia were also excluded, as were individuals with a history of surgery to the head and neck (e.g. mandible surgery).

A total of 73 adult participants, 35 males and 38 females between the ages of 18 and 88 years, from Johannesburg, SA, were recruited.

Table 1 shows that there were slightly more females than males, and most participants were in the 18 - 25-year-old age group.

The study was conducted at each participant's home, or at a public place of their convenience.

Prior to initiating the study, permission to proceed was obtained from the University of the Witwatersrand Human Research Ethics Committee (Medical) (ref. no. M171169). Thereafter a pilot study was conducted, and necessary modifications were made to the questionnaire that had been developed by the researchers for the purpose of this study. Individuals from a residential community centre were provided with information and invited to participate. Following consent and confirmation of a venue, the questionnaire was completed. The questionnaire comprised six open-ended

\begin{tabular}{|c|c|c|c|c|c|c|}
\hline \multirow[b]{2}{*}{$\begin{array}{l}\text { Age range, } \\
\text { years }\end{array}$} & \multicolumn{2}{|c|}{ Gender, $n$} & \multicolumn{4}{|c|}{ Race, $n$} \\
\hline & $\begin{array}{l}\text { Male } \\
(n=35)\end{array}$ & $\begin{array}{l}\text { Female } \\
(n=38)\end{array}$ & Black & White & Indian & Other \\
\hline $18-25(n=27)$ & 16 & 11 & 1 & 16 & 9 & 1 \\
\hline $26-35(n=5)$ & 2 & 3 & 2 & 3 & 0 & 0 \\
\hline $36-45(n=7)$ & 1 & 6 & 3 & 4 & 0 & 0 \\
\hline $46-55(n=17)$ & 9 & 8 & 2 & 14 & 1 & 0 \\
\hline $56-65(n=9)$ & 4 & 5 & 3 & 6 & 0 & 0 \\
\hline $66-75(n=4)$ & 1 & 3 & 1 & 3 & 0 & 0 \\
\hline $76-85(n=3)$ & 1 & 2 & 0 & 3 & 0 & 0 \\
\hline $86-95(n=1)$ & 1 & 0 & 0 & 1 & 0 & 0 \\
\hline
\end{tabular}

questions covering biographical information, a description of current medication, the pill regimen, a description of the size of the pills, the impact of pill size and other physical properties, and participants' experiences taking the pills. Assistance with completion was provided as required.

The aims of the pilot study were to confirm the content validity of the questionnaire, the time taken to complete it and that the terminology could be understood and was not misleading. Two participants completed the pilot study. All aims were achieved, with confirmation that the questionnaire took a minimum of 5 minutes and a maximum of 8 minutes to complete.

Thematic content analysis and descriptive statistics were used to analyse the data. Rigour was maintained by use of a reflection journal to prevent unbiased and subjective interpretations by the researcher.

Transferability of the results was achieved, as the methods used in this study can be used in similar studies with individuals of the same race and age groupings, taking the same medication.

Credibility establishes that the results of the research study are believable, regarding the quality of the results as opposed the quantity. This was achieved, as other studies on the topic confirm the findings.

Dependability was established by using a timeframe for the use of medication as an inclusion criterion, thus experiences were unlikely to change.

In terms of conformability, individuals of different ages, genders and races and from different geographical locations were used to ensure greater representation of the general population.

Informed consent, voluntary participation, confidentiality, anonymity, ensuring non-maleficence, allowing participant autonomy, being considerate of cultural variation and respect for all participants were ensured throughout the study.

\section{Results}

An overview of the types of pills taken among the sample of the population used in this study is shown in Fig. 1. The figure shows that close to half of the sample took vitamins, chronic medications and/or antibiotics. The participants who were on chronic medication took a minimum of two chronic medications daily, with a significant number taking chronic medication more than once daily. With regard to antibiotic intake, the size of the pills varied, with a maximum of $1000 \mathrm{mg}$ size noted. 


\section{The pill-swallowing experience}

When participants were asked to describe the act and process of swallowing, a range of responses were noted (Fig. 2). The majority found the process to be easy (67\%):

'I have never had any issues in swallowing tablets. The medication goes down with ease and is non-problematic.'(P61)

'I find it to be an effortless experience.' (P42)

Participants also commented that their ability to swallow pills improved with age and maturity, as well as the frequency with which they were taking them. Pill-swallowing became easier the more often they swallowed pills:

'Previously I really struggle[d] to swallow pills (up until about 13 years old) until I had to take chronic medication every day.' (P73) 'Difficult to swallow before because they were too big. I find it easy now to swallow because I am used to it.' (P6)

'I struggled taking them as a child but now take them with ease.' (P51)

One's feelings about swallowing may not always align with the emotion experienced while swallowing. Thus although the act of swallowing may in itself be easy, the emotion experienced could still be an unpleasant one. A question was therefore posed to the participants as to the emotion they would use to best describe

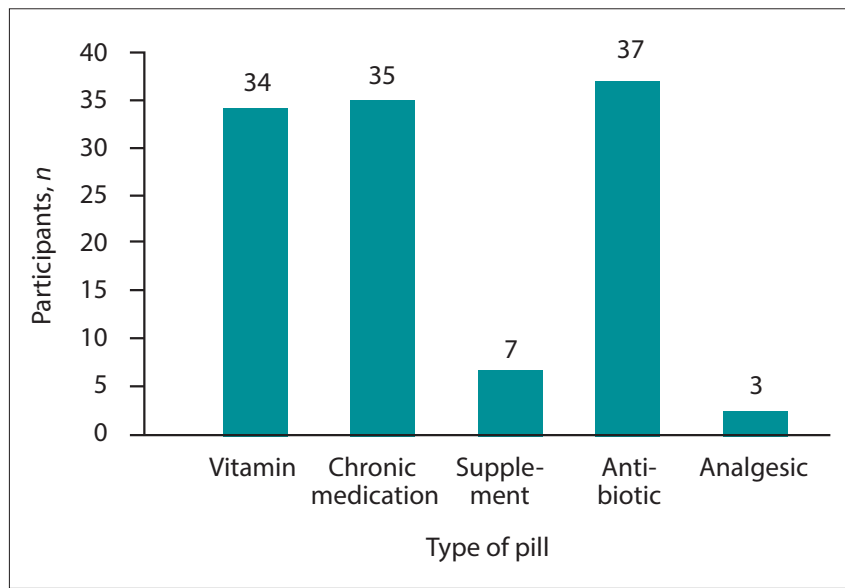

Fig. 1. Types of pills taken by participants $(\mathrm{N}=73)$.

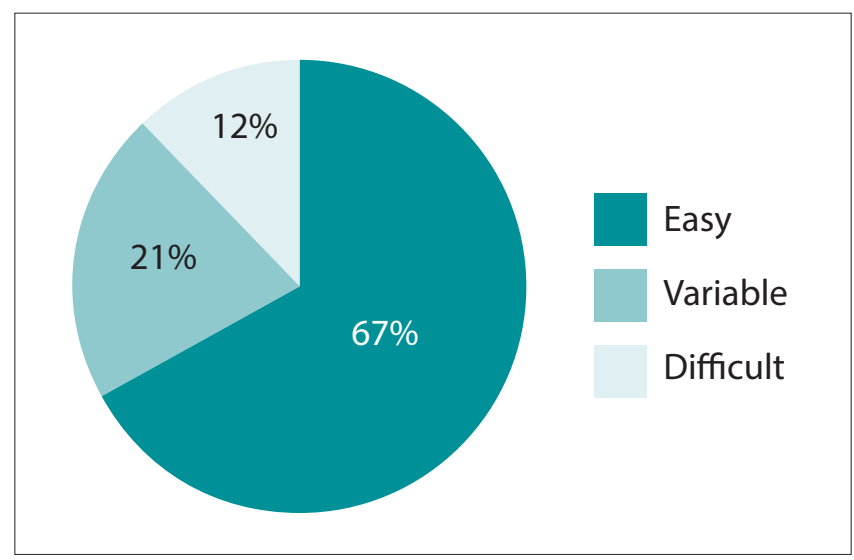

Fig. 2. Perceptions of the pill-swallowing process by participants $(\mathrm{N}=73)$. their feelings about the swallowing act. While 33\% found it to be an unpleasant experience, a larger number described the experience as good (Fig. 3). A smaller percentage of the sample said that emotion had nothing to do with it - it was a routine that needed to be completed, and so it had to be done, whether it was good or bad, pleasant or unpleasant. Therefore, these participants were ambivalent and noncommittal:

'I am not bothered. It is what it is.' (P52)

'Indifferent, occasionally uncomfortable.' (P46)

While some found it easy to swallow a pill, they still found the experience unpleasant:

'Simple procedure. I have no problem taking these pills, but I don't like taking pills long-term.'(P33)

'It's easy but not something I like to do often.'(P45)

'Terrible - tablets are taken because of prevention and because I have to.' (P26)

'It is traumatic. I prefer drinkable medication.' (P66)

It was clear that for many participants, ease of swallow did not equate to a good experience.

Gender was also noted to affect the swallowing experience. Of the $33 \%$ of the sample who found it an unpleasant experience, 78\% were female and 22\% male. Participant 54 (female) noted:

'I find it very difficult to swallow any form of pill - even the birth control, which is a tiny pill.'

\section{Changes according to the physical characteristics of the pill being taken}

It was important to establish whether the physical property of a pill affected the swallowing act, as has been documented in previous studies. Results indicated that a majority of the participants $(n=56)$ felt that the physical property of the pill did affect pill-swallowing (Fig. 4). Some participants noted that they had a more pleasurable pill-swallowing experience if they took a capsule as opposed to a hard-textured pill. Similarly, a majority of participants noted that smaller pills were easier and therefore more pleasurable to swallow than larger-sized pills.

In contrast, some participants were unaffected by the pills' physical properties. These participants did not associate emotion with pill-swallowing, and believed that if a pill needed to be taken

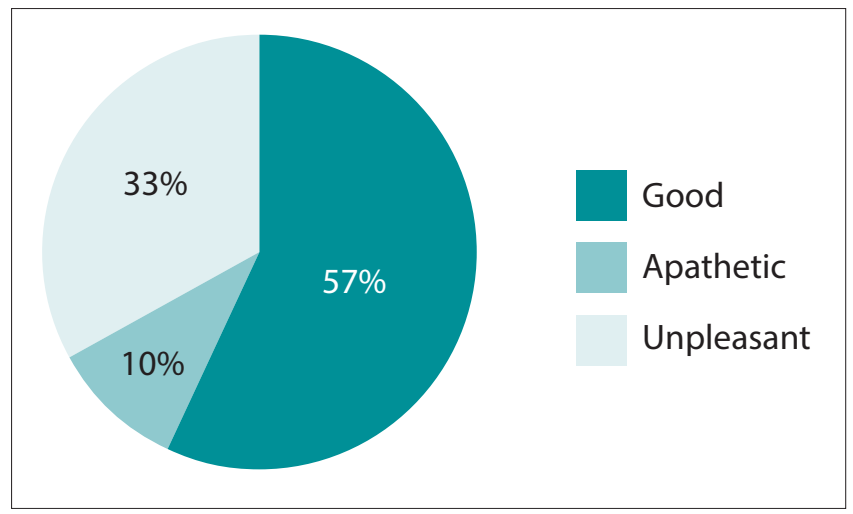

Fig. 3. Emotional association with pill-swallowing ( $N=73)$. 


\section{RESEARCH}

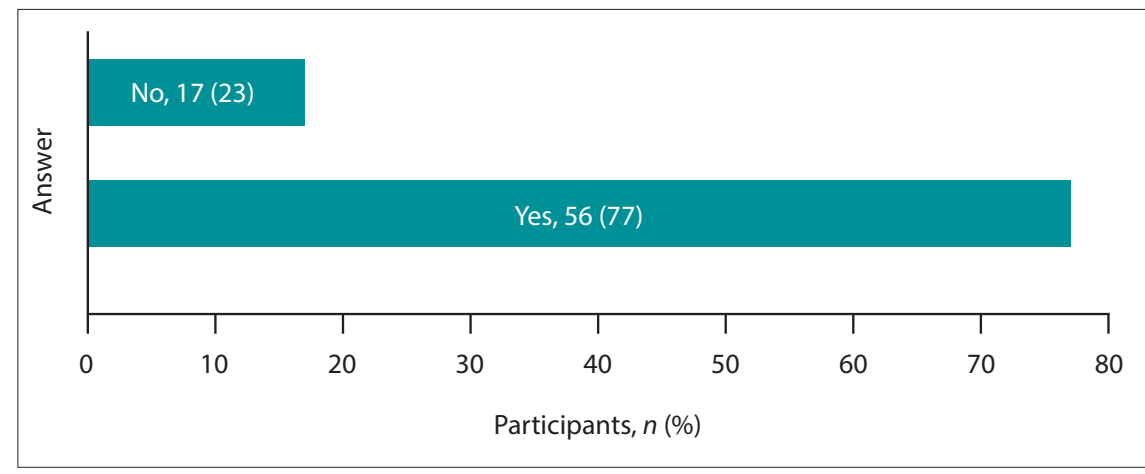

Fig. 4. Answer to 'Do the physical properties of the pill influence the pill-swallowing experience?' $(\mathrm{N}=73)$

then that had to be done regardless of size, shape or texture. This reiterates the theme of ambivalence that emerged earlier.

\section{Specific properties that positively and negatively affect the swallow- ing process}

Of the 56 participants who found that the properties of the pill influenced their ability to swallow it, Fig. 5 captures those properties that affected swallowing, from most to least influential. The properties noted in Fig. 5 were identified by the participants themselves, who often described more than one physical property as affecting swallowing. The size of the pill was identified as the biggest influence, followed by texture, taste, shape and then smell.

Thirty-eight participants found that they had difficulty swallowing 'larger, rough or bitter' pills, while they found it a lot easier to swallow smaller pills that were sugarcoated and smooth:

I find it easier to swallow small, sweet pills, they are small and delicious, so I don't feel them when I swallow.'(P66)

Participant 10 explained that rough tablets were more difficult to swallow than softer ones: 'I find taking capsules are easy and smooth or sugar-coated capsules. I can't take rough or bitter tablets.' Participant 67 agreed: 'It is easier to swallow coated capsules than it is to swallow compacted powder forms.'

Participant 22 stated: 'If it is bigger, the tablet is harder. Capsule is better. The taste seems to block it in your mouth, and you can't swallow, whereas participant 70 explained: 'Bigger tablets are harder to swallow, e.g. Puricos capsules are easier'.
With regard to shape, 11 participants found that certain pills were challenging owing to sharp ends and 'odd' shapes. Participant 51 stated: 'Zinc pills are harder to swallow as they have sharp corners and a rough texture.' Participant 28 explained how 'strange shapes (angled) are difficult to swallow.' Only 1 out of the 73 participants commented on how smell influenced his ease of swallow. Participant 48 explained how 'some medications are easier to swallow, specifically ones that really don't have a bad odour.' Given that smell was only reported as influencing one participant's swallow, this result was considered an outlier.

Taste emerged as a theme from the data. Twenty-eight participants felt that their pills tasted bitter and left them with an unpleasant aftertaste. For the purpose of this study, taste in isolation was not noted as a characteristic that resulted in difficulty in swallowing per se. This is supported by participant 30, who found it 'easy, just a little difficult when my pill has a bad taste'. Furthermore, participant 38 stated that 'the bigger pills stick in the throat; they also bitter and hard to swallow'. Finally, participant 10 explained that she 'can't take rough or bitter tablets'.

The texture of the pill was noted by 33 participants to either ease swallowing or to make it more difficult. For instance, it appeared that capsules as opposed to pills were easier to swallow, while powdered tablets were more challenging. Participant 33 stated: 'Capsules are easy and coated pills are also easy. Powdery tablets are difficult to swallow. It is likely that in addition to this being related to texture, the fact that a taste is released when a tablet disintegrates into a powder form exacerbated the difficulty.

\section{Discussion}

Pill-swallowing among individuals presenting with dysphagia is an area of concern, and is a challenge if the property of the pill cannot be changed. However, even in patients with dysphagia, despite the known difficulty of swallowing different textures, the literature documents that pills are still prescribed to them to manage underlying medical condition(s)..$^{[10]}$ Doctors grossly underestimate the pill-swallowing difficulty that may be experienced by patients, and so patients are still required to swallow oral pills. ${ }^{[1]}$ An under-researched group in SA is that of individuals who do not present with or are not diagnosed with dysphagia, but who nevertheless find pillswallowing challenging. The current study confirmed that pill swallowing is indeed a 'traumatic experience' (P66) for some individuals in general practice. This has been supported in the literature, which shows that patients may become anxious, avoid taking the pill or sometimes stop taking the pill altogether. ${ }^{[7]}$ A survey in the USA showed that $40 \%$ of adults in the general population experience challenges swallowing pills. ${ }^{[8]}$ Of this percentage, 14\% reported delaying medication consumption, and $8 \%$ skipped the dosage altogether as a result of swallowing difficulty. ${ }^{[8]}$

In contrast, some participants in the current study found that only particular properties of pills made the swallowing process difficult. For instance, size, taste and texture had adverse implications for the swallowing act. Gender was found to make a difference, in that more females than males had a negative pill-swallowing experience. This correlated with a study ${ }^{[11]}$ that found that younger patients and females were affected more often by pillswallowing than males. Schiele et al. ${ }^{[1]}$ explained these results by suggesting that it could be due to three factors: functional and anatomical differences related to age and gender; functional and structural disorders; and physical characteristics of the dosage form itself. It was also found that $54 \%$ of women aged 23 - 77 years reported difficulties in swallowing solid oral dosage forms. ${ }^{[12]}$

Shape and size affect the length of time taken for a pill to be transferred through the oesophageal phase of the swallow. 


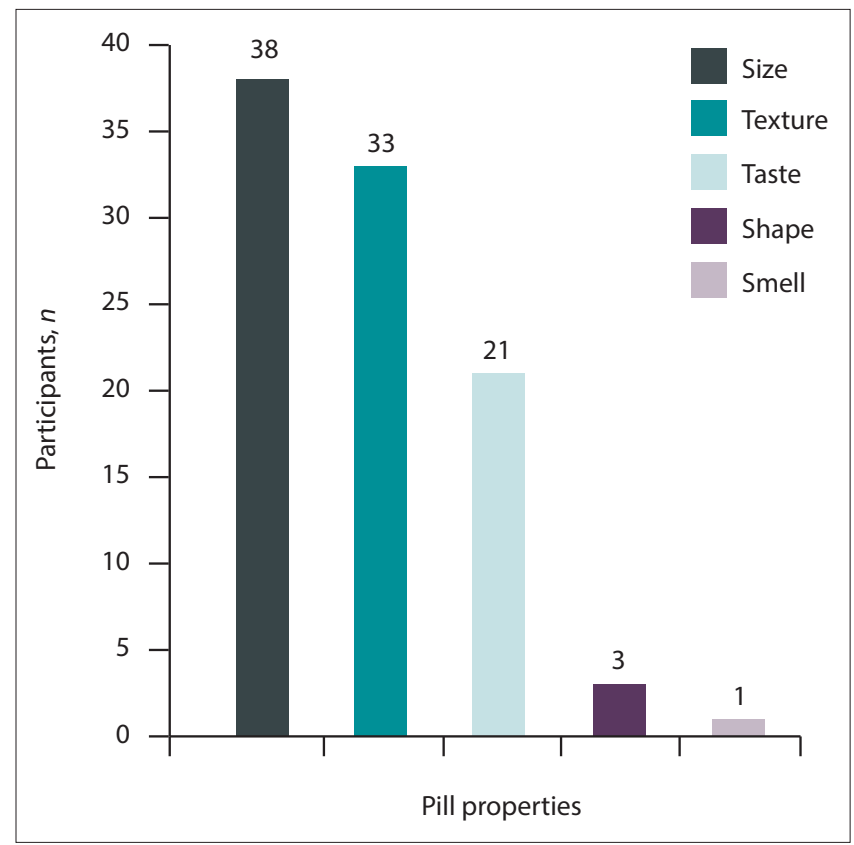

Fig. 5. Pill properties that affect the swallowing process $(\mathrm{N}=73)$.

Participants felt that smaller tablets seemed to 'go down' more quickly, and did not get stuck. These sentiments were echoed in a study by Overgaard et al. ${ }_{,}^{[13]}$ which found that increasing the size of the tablet or capsule increases the discomfort that the patient experiences when swallowing. Furthermore, it was found ${ }^{[14]}$ that smaller and oblong-shaped pills were preferred by $84 \%$ of 23 64 -year-olds, and $15 \%$ of adults aged $\geq 65$ years. This preference was similar to the results obtained in the present study, where it was noted that pills with edges seemed challenging. Older individuals seemed to prefer strongly arched and circular tablets. ${ }^{[14]}$ Participants also commented that uncoated tablets were more difficult to swallow than film-coated tablets. Coatings aid the oesophageal transit time regardless of size, as coating improves the texture of the tablet by preventing it scratching the oesophagus when passing through it. ${ }^{[13]}$ New technologies such as coatings that contain flavouring, sweeteners and gelatin (e.g. MedCoat) improve taste and transit time. ${ }^{[15]}$ This could be applied before administration in order to improve the ease of the medication intake. While only one participant mentioned smell as being influential, this may be because not many medications have a smell. ${ }^{[15]}$

Studies note that with ageing, a subtle slowing of the swallowing process occurs. ${ }^{[16]}$ Comorbidities, multiple drug use and possible reduced organ function in the elderly must also be considered when pills are prescribed. ${ }^{[17]}$ Although they were the minority of the sample, it was positive to note that some elderly participants in the current study found pills easy to swallow regardless of the physical properties.

Various compensatory strategies, such as crushing pills, breaking them into smaller parts, drinking the pills with either water or juice, or tilting one's head back when swallowing were used to ease pillswallowing. However, studies caution against ad hoc alterations to pills, namely crushing and breaking, as these may cause changes to the pharmacodynamic properties of the original dosage form. For instance, side-effects may materialise, which can range from taste alterations to fatality. ${ }^{[18,19]}$ Education for consumers of the pill, as well as more information-sharing between pharmacists and consumers, and doctors and consumers, is necessary.

Overall, in answer to the research question, regarding the pillswallowing experience of adults in general practice, the majority of participants found it to be 'pleasant and good'. However, this must be treated with caution, as some individuals also had less than optimal pill-swallowing experiences, and found it to be an unpleasant if not traumatic routine.

While attempts were made to access participants from varying geographic and socioeconomic contexts, these contexts could have been more diverse, and this is therefore a potential area for future research. Specific focus on contexts where access to water is limited, financial constraints are rife and chronic conditions exist in the midst of language barriers between doctors and those accessing medical services would be an interesting avenue of research.

\section{Conclusion}

In conclusion, the present study found that $67 \%$ of participants found the swallowing process to be easy, whereas 33\% found it to be unpleasant. Importantly, the ease of swallowing did not always correlate with the emotion attached to swallowing. To avoid challenges around pill administration and use, there is a need for input from the general population to be involved in the design of pills. For any pharmaceutical product to be used, and for the end-user to gain optimal benefit from the product, it is necessary that it is ingested in the way prescribed by the pharmaceutical company. This study highlights the need for pharmaceutical companies to continually and regularly monitor how individuals from the general population experience new pills introduced to the market. This information regarding design needs to be balanced against the pharmaceutical properties of the pill (i.e. the content) to ensure that the full benefit of the pill is realised, and that the pill-swallowing experience as a result of its physical properties does not detract from this.

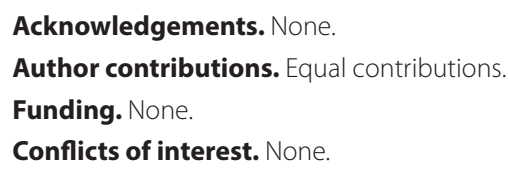

1. Bhosle M, Benner JS, DeKoven M, Shelton J. Difficult to swallow: Patient preferences for alternate valproate pharmaceutical formulations. Patient Prefer Adherence 2009;3:161171. https://doi.org/10.2147\%2Fppa.s5691

2. Ofori-Asenso R, Illomaki J, Curtis AJ, Zomer E, Zoungas S, Liew D. Patterns of medication dispensation for multiple comorbidities among older adults in Australia. Pharmacy 2018;17:E134. https://doi.org/10.3390\%2Fpharmacy6040134

3. Hales CM, Servais J, Martin CBM, Kohen D. Prescription drug use among adults aged 40 79 in the United States and Canada. National Center for Health Statistics Data Brief No 347 2019. https://www.cdc.gov/nchs/products/index.htm (accessed 13 May 2020).

4. National Ambulatory Medical Care Survey. Atlanta: Centers for Disease Control, 2017. https://www.cdc.gov/nchs/ahcd/about_ahcd.htm (accessed 13 May 2020).

5. Gilson L, Daire J. Leadership and Governance within the South African Health System. South African Health Review. Durban: Health Systems Trust, 2011.

6. Peters S, Rogers A, Salmon P, et al. What do patients choose to tell their doctors? Qualitative analysis of potential barriers to reattributing medically unexplained symptoms. J Gen Intern Med 2009;24(4):443-449. https://doi.org/10.1007\%2Fs1 1606-008-0872-x 
7. Kelly J, D'Cruz G, Wright D. A qualitative study of the problems surrounding medicine administration to patients with dysphagia. Dysphagia 2009:24(1):49-56. https://do org/10.1007/s00455-008-9170-3

8. Carnaby-Mann G, Crary M. Pill swallowing by adults with dysphagia. Arch Otolaryngo Head Neck Surg 2005:131(11): 970-975. https://doi.org/10.1001/archotol.131.11.970

9. Stegemann S, Ecker F, Maio M, Kraahs P, Wohlfart R, Breitkreutz J. Geriatric drug therapy: Neglecting the inevitable majority. Ageing Res Rev 2010;9(4):384-398. https://doi. org/10.1016/j.arr.2010.04.005

10. Kaplan BJ, Steiger RA, Pope J, Marsh A, Sharp M, Crawford SG. Successful treatment of pillswallowing difficulties with head posture practice. Paediatr Child Health 2010;15:e1-e5. https://doi.org/10.1093/pch/15.5.e

11. Schiele JT, Quinzler R, Klimm HD, Pruszydlo MG, Haefeli WE. Difficulties swallowing oral dosage forms in a general practice population: Prevalence, causes, and relationships to dosage forms. Eur J Clin Pharmacol 2013; 69(4):937-948. https://doi.org/10.1007/s00228012-1417-0

12. Fields J, Go J, Schulz K. Pill properties that cause dysphagia and treatment failure. Curr Ther Res 2015;77:79-82. https://doi.org/10.1016\%2Fj.curtheres.2015.08.002

13. Overgaard A, Møller-Sonnergaard J, Christrup L, Højsted J, Hansen R. Patient's evaluation of shape, size, and colour of solid dosage forms. Pharm World Sci 2001;23(5):185-188. https:// doi.org/10.1023/a:1012050931018
14. Stegemann S, Gosch M, Breitkreutz J. Swallowing dysfunction and dysphagia is an unrecognized challenge for oral drug therapy. Int J Pharmaceutics 2012:430(1-2):197-206. https://doi.org/10.1016/j.jpharm.2012.04.022

15. Liu F, Ranmal S, Batchelor H, Orlu-Gul M, Ernest T, Thomas I. Patient-centered pharmaceutical design to improve acceptability of medicines: Similarities and differences in paediatric and geriatric populations. Drugs 2014;74(16):1871-1889. https://doi org/10.1007/s40265-014-0297-2

16. Sura L, Madhavan A, Carnaby G, Crary MA. Dysphagia in the elderly: Management and nutritional consideration. Clin Interv Aging 2012;7:287-298. https://doi. org/10.2147\%2FCIA.S23404

17. Breitkreutz J, Boos J. Paediatric and geriatric drug delivery. Expert Opin Drug Deliv 2007;4(1):37-45. https://doi.org/10.1517/17425247.4.1.37

18. Bourdenet G, Saussereau E, Goulle JP, Guerbet M, Doucet J. Crushing drugs in geriatric units: Are cleaning methods and devices appropriate? Eur J Clin Pharm 2018:75(1):127129. https://doi.org/10.1007/s00228-018-2547-9

19. Schier JG, Howland MA, Hoffman, Nelson LS. Fatality from administration of labetalol and crushed extended-release nifedipine. Ann Pharmacother 2003;37(10):1420-1423. https:// doi.org/10.1345/aph.1d091

Accepted 18 June 2020 